\title{
Measuring parasite fitness under genetic and thermal variation
}

\author{
PF Vale and TJ Little \\ Institute of Evolutionary Biology, School of Biological Sciences, University of Edinburgh, Ashworth Labs, Edinburgh, Scotland, UK
}

\begin{abstract}
Accurate measures of parasite fitness are essential to study host-parasite evolution. Parasite fitness depends on several traits involved in establishing infection, growth and transmission. Individually, these traits provide a reasonable approximation of fitness, but they may also be under the shared control of both host and parasite genetics $\left(G_{H} \times G_{P}\right.$ interactions), or be differentially sensitive to environmental variation. Using the natural host-parasite system Daphnia magna-Pasteuria ramosa, we performed experimental infections that incorporated host and parasite genetic variation at three different temperatures, and compared the measures of parasite fitness based only on growth rate, or incorporating the ability to infect. We found that infectivity was most important for parasite fitness and depended
\end{abstract}

mainly on the combination of host and parasite genotypes. Variation in post-infection parasite growth and killing time depended on the parasite genotype and its interaction with temperature. These results highlight the merits of studies that can incorporate natural infection routes and emphasize that accurate measures of parasite fitness require knowledge of the genetic control and environmental sensitivity of more than one trait. In addition, no $G_{H} \times G_{P} \times E$ interactions were present, suggesting that the potential for genetic specificities to drive frequency-dependent coevolution in this system is robust to thermal variation.

Heredity (2009) 103, 102-109; doi:10.1038/hdy.2009.54; published online 20 May 2009

Keywords: parasite fitness; genotype-by-environment interaction; genotype-by-genotype interaction; temperature; infection; transmission

\section{Introduction}

Parasite growth has commonly been used as a parasite fitness correlate, particularly in empirical work testing the evolution of virulence theory (for example, Read and Schrag, 1991; Ebert, 1998; Jensen et al., 2006). Models of coevolutionary interactions, however, make the important point that the host and parasite genotype will together determine virulence, as well as the probability of becoming infected (reviewed in Lambrechts et al., 2006b). Empirical work motivated by this coevolutionary perspective has shown that infection outcomes, and ultimately the fitness of both host and parasite, can indeed depend strongly on particular combinations of host and parasite genotypes, called $\mathrm{G}_{\mathrm{H}} \times \mathrm{G}_{\mathrm{P}}$ interactions (for example, Carius et al., 2001; Lambrechts et al., 2005; Lazzaro et al., 2006).

In addition to the genetic context, infection outcomes may depend on the abiotic environment. Laboratory studies designed to tease apart genetic effects on infection outcomes tend to minimize environmental variation but such variation are widespread in the wild and could affect genotypes differently, leading to genotype-by-environment interactions $(\mathrm{G} \times \mathrm{E}$, reviewed in Lazzaro and Little, 2008). For example, genotype-byfood level interactions have been found to have strong

Correspondence: Dr PF Vale, School of Biological Sciences, Institute of Evolutionary Biology, University of Edinburgh, Ashworth Labs, Wesrt Mains Road, Edinburgh, Scotland EH9 3JT, UK.

E-mail: pedro.vale@ed.ac.uk

Received 19 January 2009; revised 7 April 2009; accepted 7 April 2009; published online 20 May 2009 effects on infection outcomes in mosquito-Plasmodium systems (Ferguson and Read, 2002; Lambrechts et al., 2006a), a mosquito-microsporidian system (Bedhomme et al., 2004) and during bacterial infection of a ciliate (Restif and Kaltz, 2006). Experimental variation in temperature has uncovered $\mathrm{G} \times \mathrm{E}$ interactions affecting infection outcomes in the pea aphid-fungal system Acyrthosiphon-Erynia (Blanford et al., 2003) and the waterflea-bacterial system Daphnia-Pasteuria (Mitchell et al., 2005).

These interactions between genotypes or between genotypes and the environment could result in host and parasite genotypes having context-dependent fitness (Lambrechts et al., 2006b; Lazzaro and Little, 2008), with implications for the maintenance of genetic variation in the wild. $G_{H} \times G_{P}$ interactions, for example, can generate frequency-dependent selection, which prevents the loss of rare alleles and fosters coevolution between host and parasite populations (Hamilton, 1993). Alternatively, $\mathrm{G} \times \mathrm{E}$ interactions can maintain genetic variation when alternate genotypes are favoured in different environments (reviewed in Byers, 2005; Laine and Tellier, 2008). $\mathrm{G}_{\mathrm{H}} \times \mathrm{G}_{\mathrm{P}} \times \mathrm{E}$ interactions could favour different combinations of coevolving genotypes in different environments or generate different rates of coevolution between hosts and parasites in different environments (Thompson, 1994, 1999), further shaping the temporal and spatial distribution of genetic diversity and patterns of local adaptation (Kaltz and Shykoff, 1998; Laine, 2008). In all these cases, a correct assessment of whether environmental variability can maintain polymorphism clearly relies on an accurate measurement of fitness. Although 
parasite growth or transmission potential is a commonly used (and reasonable) parasite fitness correlate, a complete assessment of fitness must incorporate all stages of infection, as a parasite must infect, grow and transmit to new hosts. Each of these traits may be under differential genetic (host or parasite) and environmental control.

Earlier studies of the host-parasite system used presently, the crustacean Daphnia magna and its specialist bacterium Pasteuria ramosa, have found that temperature is an important determinant of infection and a potential driver of epidemics in wild populations (Mitchell et al., 2004; Duncan and Little, 2007). Experimental studies in this system have found evidence for host genotype $\times$ temperature interactions $\left(\mathrm{G}_{\mathrm{H}} \times \mathrm{E}_{\mathrm{T}}\right)$ affecting the probability of infections becoming established (Mitchell et al., 2005), parasite genotype $\times$ temperature interactions $\left(\mathrm{G}_{\mathrm{P}} \times \mathrm{E}_{\mathrm{T}}\right)$ affecting parasite growth (Vale et al., 2008), as well as strong $G_{H} \times G_{P}$ interactions for infectivity (Carius et al., 2001). Given that Daphnia inhabits temperate ponds where temperature fluctuates on a seasonal or even daily scale, studying these interactions can inform whether evolutionary or coevolutionary trajectories are sensitive to environmental variation. We now extend the study of interactions with an experiment simultaneously incorporating host genetic variation, parasite genetic variation and temperature variation. We measured the probability of a host becoming infected, and post-infection host mortality and parasite growth at 15,20 and $25^{\circ} \mathrm{C}$. In doing so, we examine which infection traits are more sensitive to thermal variation and important in determining parasite fitness. This design also allowed us to assess if previously described genetic specificities (that is, $\mathrm{G}_{\mathrm{H}} \times \mathrm{G}_{\mathrm{P}}$ interactions) can be altered under thermal variation $\left(G_{H} \times G_{P} \times E_{T}\right.$ interaction).

\section{Materials and methods}

\section{Host and parasite isolates}

The experiment used long-term laboratory isolates of hosts and parasites. All host genotypes (GG3, GG4, GG7 and GG13) and parasite isolates (Sp1, Sp7, Sp8 and Sp13) were originally collected from a German population near Gaarzerfeld, Germany, in a study showing that infection outcomes depend on the specific combination of host and parasite genotype (Carius et al., 2001). Since this time, host clones have been maintained in the lab in a state of clonal reproduction and parasite isolates have been kept frozen except for occasional experimental use. Daphnia are filter feeders and become infected with $P$. ramosa by filtering transmission spores present in the water. Within the host, $P$. ramosa goes through a slow developmental process, culminating in the formation of transmission-stage spores, apparent 15-20 days postinfection at $20^{\circ} \mathrm{C}$. Transmission is exclusively horizontal, achieved by spores that are released from dead hosts. During the infection process, infected female D. magna become sterilized and ultimately die prematurely (Ebert et al., 1996).

\section{Infections and temperature treatments}

Before infection, host maternal lines were acclimatized for three generations to equilibrate maternal and envi- ronmental effects. Twelve replicate jars of each isofemale line (five Daphnia per jar) were contained in $200 \mathrm{ml}$ of artificial medium (Kluttgen et al., 1994), fed $6 \times 10^{6}$ cells per Daphnia per day of chemostat-grown Scenedesmus obliquus algae and maintained within temperaturecontrolled incubators with a light/dark cycle of 12:12 h. Medium was changed with every clutch or every 3-4 days regardless of a clutch being present. Although infections were carried out at three different temperatures, all host lines were acclimatized at $20^{\circ} \mathrm{C}$. Earlier experiments have shown that the temperature of acclimation of the maternal generation does not affect susceptibility to infection in their offspring (Mitchell and Read, 2005). Acclimation at the same temperature allows clutch production to be synchronized and infections to be performed on the same day. After acclimation, infections were carried out at 15,20 and $25^{\circ} \mathrm{C}$ by splitting replicates among three incubators at these temperatures. Incubators do not vary substantially apart from the temperature at which they are set; earlier experiments (for example, Mitchell et al., 2005) have shown that host phenotypes are consistent with the temperature treatment, regardless of which incubator they were studied in.

Infections followed a split-jar design (analogous to a split-brood design), where clutches from each replicate jar of each host genotype were split into the different treatments (four parasite genotype exposures at three temperatures). Each experimental replicate consisted of a single, 1-day-old $(<24 \mathrm{~h})$ female, placed in a jar containing $60 \mathrm{ml}$ of artificial Daphnia medium and sterile sand. These jars were placed in an incubator at the appropriate temperature overnight before the beginning of infection to guarantee that the entire infection period occurred at the desired temperature. All hosts were exposed to 10000 parasite spores per jar. Spore suspensions were originally obtained by homogenizing infected Daphnia, and these suspensions were stored at $-20^{\circ} \mathrm{C}$ until required. Before infection, spore suspensions were thawed, shaken thoroughly and counted using a Neubauer counting chamber $\left(0.0025 \mathrm{~mm}^{2} \times 0.1 \mathrm{~mm}\right.$ depth) (Hawksley, Sussex, UK). Daphnia have longer development times and slower filtration rates at lower temperatures (Peters and De Bernardi, 1987), so a measure of Daphnia physiological time is useful. Degree-day is a reasonable measure of Daphnia physiological time and is simply the product of temperature and real days, with Daphnia producing the first clutch after 250-280 degree-days across a range of temperatures (Mitchell et al., 2005). Following previous temperature manipulation studies in this system (Mitchell et al., 2005; Vale et al., 2008), Daphnia were exposed to parasites for 150 degree-days, that is, 6 days at $25^{\circ} \mathrm{C}, 7.5$ days at $20^{\circ} \mathrm{C}$ and 10 days at $15^{\circ} \mathrm{C}$. During the infection period, all replicates were stirred daily and fed low amounts of chemostat-grown $S$. obliquus algae $\left(1.5 \times 10^{6}\right.$ cells per Daphnia). The combination of stirring and low food increases the likelihood of Daphnia encountering transmission spores and becoming infected.

After the infection period, all replicates were transferred to jars with $60 \mathrm{ml}$ clean medium, and following the procedure of Mitchell et al., (2005), food levels were increased to $2 \times 10^{6}$ at $15^{\circ} \mathrm{C}, 3.5 \times 10^{6}$ at $20^{\circ} \mathrm{C}$ and $6 \times 10^{\circ}$ at $25^{\circ} \mathrm{C}$ to be in excess of what the Daphnia can consume, but not so high as to induce mortality. From degree-day 
300-500, hosts were observed under a dissecting microscope for symptoms consistent with $P$. ramosa infection (sterilization and bacterial growth in the haemolymph). Jars were checked daily for dead hosts and these were removed from the jars as soon as detected, individually placed in 1.5-ml Eppendorf tubes and frozen at $-20^{\circ} \mathrm{C}$. The experiment lasted 900 degree-days and, at this time, all remaining Daphnia were individually frozen. Counts of $P$. ramosa transmission-stage spores were obtained by crushing the dead host with a sterile plastic pestle and counting two independent samples of this suspension in a Neubauer (improved) counting chamber $\left(0.0025 \mathrm{~mm}^{2} \times 0.1 \mathrm{~mm}\right.$ depth). The number of transmission spores was used as a measure of transmission potential. Offspring production was not recorded, as this experiment focused on parasite fitness traits. Throughout the experiment, jars were distributed randomly within trays of 24 jars and the position of the trays was changed regularly to equilibrate any positional effects within the incubators.

\section{Data analysis}

Analyses were performed using the statistical software packages JMP 7 (SAS Institute Inc., Cary, NC, USA) and R (R, 2005, Vienna, Austria). We tested fixed effects of host genotype, parasite genotype and temperature on infectivity (the proportion of hosts infected), host mortality (mean time to host death) and parasite transmission potential as estimated by counting transmission spores. For infectivity, we used a generalized linear model with a binomial error structure and a logit function. Host survival (the time to host death) was analysed for infected individuals only, using proportional hazards. The time scale used was always degreeday to allow comparisons among temperature treatments, and all individuals that were alive by the end of the experiment were entered as censored data. Spore counts were square-root-transformed to obtain normally distributed data and were analysed using analysis of variance.

The number of spores produced per infected host was analysed, first, by counting the spores produced by an individual infection, irrespective of whether that individual died during the experimental period or survived until the end. Variation in this measure can thus be attributed either to different rates of parasite growth or to differential host survival rates, but by incorporating both factors, this represents the lifetime transmission potential of an infection (see Jensen et al., 2006). Second, we controlled for variation due to differential survival by analysing the number of spores produced per degreeday a host remained alive (that is, spores per host per degree-day). This measure represents, roughly, parasite growth rate.

The above analyses of spore counts were restricted to infected individuals. However, we also considered that failure to infect is extremely detrimental for parasite fitness. We, therefore, studied the lifetime transmission potential of each parasite genotype on all hosts, regardless of the infection status (hosts that did not become infected contributed zero spores and thus parasite genotypes produce, on average, a number of spores that are weighted by their infectivity on all hosts). Given that many host-parasite combinations yielded unsuccessful infections, this resulted in a high number of data points to be zero. To correct for the resulting overdispersion of the data, we used generalized linear models with both a Poisson and a quasi-Poisson error structure in $R$ (Crawley, 2007). However, we found the results from these to be essentially identical to the linear model, and we therefore present only the latter for consistency.

Although the significance of each term was determined based on the reduced model, we also report the proportion of variance explained by each of the terms in the full model. For infectivity, the deviance of each term was divided by the total deviance of the binomial model mentioned above. For spore-production data, variance proportions were calculated for each term as their sequential sum of squares divided by the total sum of squares in the model. For survival, to enable straightforward calculation of the effect sizes of each term, we analysed 'day of death' using analysis of variance and calculated variance as for spore production. The reduced model in both analysis of variance and formal survival analysis (proportional hazards) did not yield different qualitative results.

\section{Results}

We exposed a total of 576 individual Daphnia to infection (12 replicates of four host genotypes exposed to four parasite genotypes at three temperatures), of which 38 replicates were lost during the experiment, mainly due to death during the infection period. Owing to the small size of hosts and early stage of infection, it was not possible to determine why these replicates died, so we removed them from all subsequent analyses. Most of the treatments remained with a total of 12 replicates, with the lowest number of replicates being nine for three of the $25^{\circ} \mathrm{C}$ treatments.

Across all combinations of host and parasite genotypes, infectivity was highest at $20^{\circ} \mathrm{C}(33 \%)$, and lower at $15^{\circ} \mathrm{C}(18 \%)$ and at $25^{\circ} \mathrm{C}(16 \%)$ (Figure 1$)$. The reduced model (Table 1) shows that the proportion of hosts that became infected can be explained mostly by main effects of temperature and host genotype. These main effects explained over $25 \%$ of the variance. There was a significant interaction between host genotype and parasite genotype, explaining nearly $13 \%$ of the variance, and also a significant interaction between host genotype and temperature, but this effect explained less than $3 \%$ of the variance (Table 1). The three-way interaction was not significant in the full model $\left(\chi_{18}^{2}=8.271, P=0.974\right)$.

Temperature influenced the survival of infected hosts, explaining $23 \%$ of the variation in the time to death (Table 1; Figure 2a). The direction and magnitude of this effect depended on the parasite genotype that was involved in the infection. This parasite genotype-bytemperature interaction explained $8 \%$ of the variation for the time to host death (Table 1; Figure 2b). In some cases, we observed a complete switch in the rank order of parasite genotypes between temperatures (Figure 2b). Notably, none of the hosts infected with parasite genotype Sp8 died during the experiment at $20^{\circ} \mathrm{C}$, but this parasite genotype is the most virulent at $25^{\circ} \mathrm{C}$, killing all hosts it infected by degree-day 600 (24 days).

The lifetime transmission potential of parasites could be explained by temperature, and also by an interaction between parasite genotype and temperature (nearly $30 \%$ 


\begin{tabular}{|c|c|c|c|c|c|}
\hline \multicolumn{7}{|c|}{$15^{\circ} \mathrm{C}$} \\
\hline & GG3 & GG4 & GG7 & GG13 & Mean \\
\hline Sp1 & $\mathbf{0 . 7 5}$ & $\mathbf{0 . 8 2}$ & 0.00 & 0.00 & 0.39 \\
\hline Sp13 & 0.27 & 0.00 & 0.00 & 0.25 & 0.13 \\
\hline Sp7 & 0.25 & 0.25 & 0.00 & 0.00 & 0.13 \\
\hline Sp8 & 0.17 & 0.00 & 0.00 & 0.08 & 0.06 \\
\hline Mean & 0.36 & 0.27 & 0.00 & 0.08 & 0.18 \\
\hline
\end{tabular}

\begin{tabular}{|c|c|c|c|c|c|}
\hline & GG3 & GG4 & GG7 & GG13 & Mean \\
\cline { 1 - 5 } Sp1 & $\mathbf{1 . 0 0}$ & $\mathbf{1 . 0 0}$ & 0.08 & 0.00 & 0.52 \\
\cline { 1 - 5 } Sp13 & $\mathbf{0 . 7 5}$ & 0.00 & 0.09 & 0.25 & 0.27 \\
\cline { 1 - 4 } Sp7 & 0.67 & 0.17 & 0.20 & 0.00 & 0.26 \\
\hline Sp8 & $\mathbf{0 . 7 5}$ & 0.08 & 0.00 & 0.20 & 0.26 \\
\hline Mean & 0.79 & 0.31 & 0.09 & 0.11 & 0.33 \\
\hline
\end{tabular}

\begin{tabular}{|c|c|c|c|c|c|}
\hline & GG3 & GG4 & GG7 & GG13 & Mean \\
\hline Sp1 & $\mathbf{0 . 7 0}$ & $\mathbf{0 . 8 2}$ & 0.00 & 0.00 & 0.38 \\
\hline Sp13 & 0.09 & 0.10 & 0.00 & 0.00 & 0.05 \\
\hline Sp7 & 0.42 & 0.18 & 0.09 & 0.00 & 0.17 \\
\hline Sp8 & 0.00 & 0.08 & 0.00 & 0.00 & 0.02 \\
\hline Mean & 0.30 & 0.30 & 0.02 & 0.00 & 0.16 \\
\hline
\end{tabular}

Figure 1 Infectivity across temperatures. The matrices show the proportion infected for each combination of host (columns) and parasite (rows) genotypes. Numbers are the proportion of 12 individual replicates that became infected. Combinations of host and parasite genotypes with higher infectivity are darker compared with combinations with little or no infectivity. See Table 1 for statistical details.

Table 1 Summary of statistical analyses testing the effects of temperature, host and parasite genotypes on infectivity and host survival

\begin{tabular}{|c|c|c|c|c|}
\hline Source & d.f. & $L-R \chi^{2}$ & P-value & $\%$ Variance full model \\
\hline \multicolumn{5}{|l|}{ Infectivity } \\
\hline Host & 3 & 104.9 & $<0.0001$ & 20.69 \\
\hline Parasite & 3 & 2.147 & 0.5425 & 10.79 \\
\hline Temperature & 2 & 28.21 & $<0.0001$ & 4.91 \\
\hline Host $\times$ Parasite & 9 & 62.28 & $<0.0001$ & 12.58 \\
\hline Host $\times$ Temperature & 6 & 13.56 & 0.0350 & 2.82 \\
\hline Parasite $\times$ Temperature & 6 & 8.68 & 0.1924 & 1.61 \\
\hline Host $\times$ Temperature $\times$ Parasite & - & - & NS & 1.54 \\
\hline Error & 490 & & & 45.06 \\
\hline Total & 537 & & & 100 \\
\hline \multicolumn{5}{|l|}{ Survival among infected hosts } \\
\hline Host & 3 & 2.20 & 0.5328 & 3.87 \\
\hline Parasite & 3 & 4.93 & 0.1772 & 4.61 \\
\hline Temperature & 2 & 13.69 & 0.0011 & 23.14 \\
\hline Host $\times$ Parasite & - & - & NS & 5.15 \\
\hline Host $\times$ Temperature & - & - & NS & 2.13 \\
\hline Parasite $\times$ Temperature & 6 & 14.30 & 0.0265 & 8.10 \\
\hline Host $\times$ Parasite $\times$ Temperature & - & - & NS & 0.93 \\
\hline Error & 93 & & & 52.07 \\
\hline Total & 120 & & & 100 \\
\hline
\end{tabular}

Abbreviation: NS, not significant.

Infectivity was modelled using a generalized linear model (GLM), assuming a binomial error structure. Survival was analysed with proportional hazards analysis on infected individuals only. We present the test statistics for all the terms in the reduced model. \% Variance explained by each term refers to the full model. d.f. is the degrees of freedom for the reduced model. L-R $\chi^{2}$ is the likelihood ratio $\chi^{2}$. Terms are significant for $P>0.05$. See Materials and methods section for statistical details.
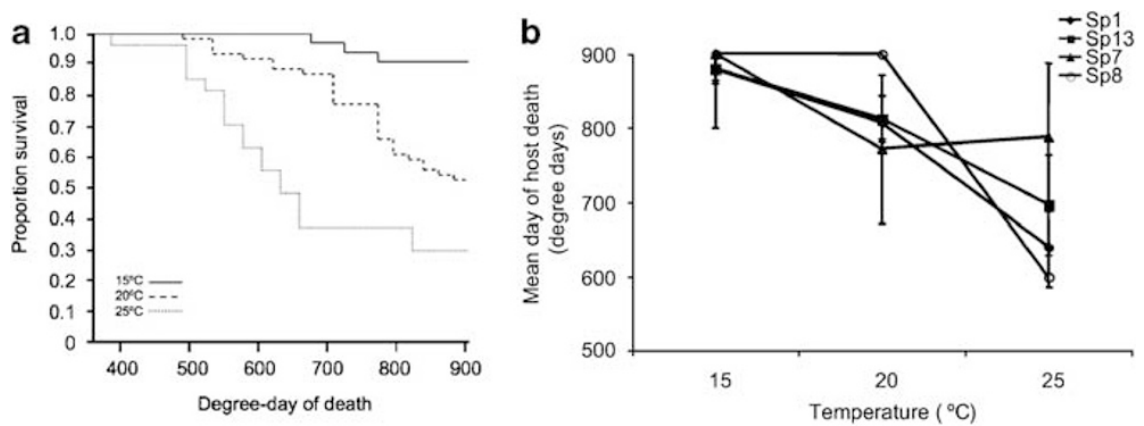

Figure 2 Host survival under thermal variation. (a) Survival curves for infected hosts at $15{ }^{\circ} \mathrm{C}$ (full black line), $20^{\circ} \mathrm{C}$ (dashed line) and $25^{\circ} \mathrm{C}$ (dotted line). (b) Reaction norms for the mean time to host death in infected hosts. Each data point is the mean trait value for each parasite genotype. Error bars are standard error of the mean. See Table 1 for statistical details.

of the variance, combined; Table 2). Host genotype had no effect on lifetime transmission potential, suggesting that within-host growth is only controlled by the parasite genotype and is conditioned by the temperature it experiences (Figure 3a). Removing host genotype as a main effect did not improve the overall model fit. After controlling for differential survival (by studying spores per Daphnia per degree-day, which is a measure of parasite growth rate), we found that the parasite $\times$ temperature interaction was no longer sig- 
nificant, and variation in spore production could be explained to a large extent by significant main effects of temperature (39\% variance explained) and parasite

Table 2 Summary of statistical analyses testing the effects of temperature, host and parasite genotypes on different measures of parasite growth

\begin{tabular}{|c|c|c|c|c|c|}
\hline \multirow[b]{2}{*}{ Lifetime transmission potential } & \multicolumn{3}{|c|}{ Reduced model } & \multicolumn{2}{|c|}{ Full model } \\
\hline & & & & & \\
\hline Source & d.f. & $\mathrm{F}$ ratio & $\mathrm{F}$ & d.f. & $\%$ Var \\
\hline Host & 3 & 0.8825 & 0.453 & 3 & 1.61 \\
\hline Parasite & 3 & 1.9871 & 0.120 & 3 & 3.62 \\
\hline Temperature & 2 & 18.1799 & $<0.0001$ & 2 & 22.10 \\
\hline Host $\times$ Parasite & - & - & NS & 6 & 2.42 \\
\hline Host $\times$ Temperature & - & - & NS & 4 & 1.23 \\
\hline Parasite $\times$ Temperature & 6 & 2.258 & 0.043 & 6 & 7.75 \\
\hline Host $\times$ Parasite $\times$ Temperature & - & - & NS & 3 & 1.93 \\
\hline Model & & & & 27 & 40.66 \\
\hline Error & & & & 93 & 59.34 \\
\hline Total & & & & 120 & 100 \\
\hline \multicolumn{6}{|l|}{ Parasite growth rate } \\
\hline Host & 3 & 1.0747 & 0.363 & 3 & 1.60 \\
\hline Parasite & 3 & 2.1579 & 0.097 & 3 & 3.20 \\
\hline Temperature & 2 & 39.3594 & $<0.0001$ & 2 & 38.95 \\
\hline Host $\times$ Parasite & - & - & NS & 6 & 1.44 \\
\hline Host $\times$ Temperature & - & - & NS & 4 & 0.88 \\
\hline Parasite $\times$ Temperature & 6 & 1.2771 & 0.274 & 6 & 3.53 \\
\hline Host $\times$ Parasite $\times$ Temperature & - & - & NS & 3 & 1.06 \\
\hline Model & & & & 27 & 50.66 \\
\hline Error & & & & 93 & 49.34 \\
\hline Total & & & & 120 & 100 \\
\hline \multicolumn{6}{|c|}{ Spores on all hosts (infected +not infected) } \\
\hline Host & 3 & 53.211 & $<0.0001$ & 3 & 15.63 \\
\hline Parasite & 3 & 30.3754 & $<0.0001$ & 3 & 8.92 \\
\hline Temperature & 2 & 30.1396 & $<0.0001$ & 2 & 5.90 \\
\hline Host $\times$ Parasite & 9 & 15.9416 & $<0.0001$ & 9 & 14.05 \\
\hline Host $\times$ Temperature & 6 & 9.0014 & $<0.0001$ & 6 & 5.29 \\
\hline Parasite $\times$ Temperature & - & - & NS & 6 & 0.44 \\
\hline Host $\times$ Parasite $\times$ Temperature & - & - & NS & 18 & 2.36 \\
\hline Model & & & & 47 & 52.60 \\
\hline Error & & & & 490 & 47.40 \\
\hline Total & & & & 537 & 100 \\
\hline
\end{tabular}

Abbreviation: NS, not significant.

We present the test statistics for all the terms in the reduced model. $\%$ Variance explained by each term refers to the full model. d.f., degrees of freedom for the reduced model. $F$ is the F-ratio test statistic. Terms are significant for $P>0.05$. See Materials and methods section for statistical details. genotype (3.2\% variance explained, Table 2$)$. Incorporating information on the infectivity of each parasite genotype also dissipated the parasite genotype-bytemperature interaction (Table 2; Figure 3b).

\section{Discussion}

A growing body of empirical work, mostly on plant and invertebrate host-pathogen systems, has shown that infection is frequently dependent on the host and parasite genotypes involved $\left(\mathrm{G}_{\mathrm{H}} \times \mathrm{G}_{\mathrm{P}}\right)$ (Thompson and Burdon, 1992; Carius et al., 2001; Lambrechts et al., 2006b; Salvaudon et al., 2007) and on the environmental context (E) in which host and parasite genotypes interact $\left(\mathrm{G}_{\mathrm{H}} \times \mathrm{E}, \mathrm{G}_{\mathrm{P}} \times \mathrm{E}\right.$ and $\mathrm{G}_{\mathrm{H}} \times \mathrm{G}_{\mathrm{P}} \times \mathrm{E}$, for example, Mitchell et al., 2005; Laine, 2007b; reviewed in Lazzaro and Little, 2008). Here, we tested whether simultaneous variation in temperature $\left(\mathrm{E}_{\mathrm{T}}\right)$, and host and parasite genetic background, could modify infection outcomes when the crustacean D. magna is exposed to the bacterium P. ramosa. Our experiment included four genotypes of the host and four genotypes of the parasite that were earlier established to show substantial differences in infectionrelated traits as well as genetic specificity for infectivity $\left(G_{H} \times G_{P}\right.$ interactions; Carius et al., 2001). This genetic specificity was confirmed, but we also uncovered a set of genotype-by-environment $(\mathrm{G} \times \mathrm{E})$ interactions. A host genotype-by-environment interaction $\left(\mathrm{G}_{\mathrm{H}} \times \mathrm{E}_{\mathrm{T}}\right)$ was present for the probability of becoming infected, but not for traits that were measured later in the infection process. By contrast, parasite genotype-by-environment interactions $\left(\mathrm{G}_{\mathrm{P}} \times \mathrm{E}_{\mathrm{T}}\right)$ were not evident for the probability of becoming infected, but were important for traits (transmission potential and the mortality of infected hosts) that were relevant once infections were established. Threeway, $\mathrm{G}_{\mathrm{H}} \times \mathrm{G}_{\mathrm{P}} \times \mathrm{E}$, interactions were not evident.

\section{Infectivity}

The $\mathrm{G}_{\mathrm{H}} \times \mathrm{E}_{\mathrm{T}}$ for infectivity is not attributable to changes in the relative rank order of host and parasite genotypes across temperatures, but is instead associated with changes in the magnitude of differences in infectivity between temperature treatments for different host genotypes (Figure 1). Although both scenarios will influence the strength of selection, the long-term maintenance
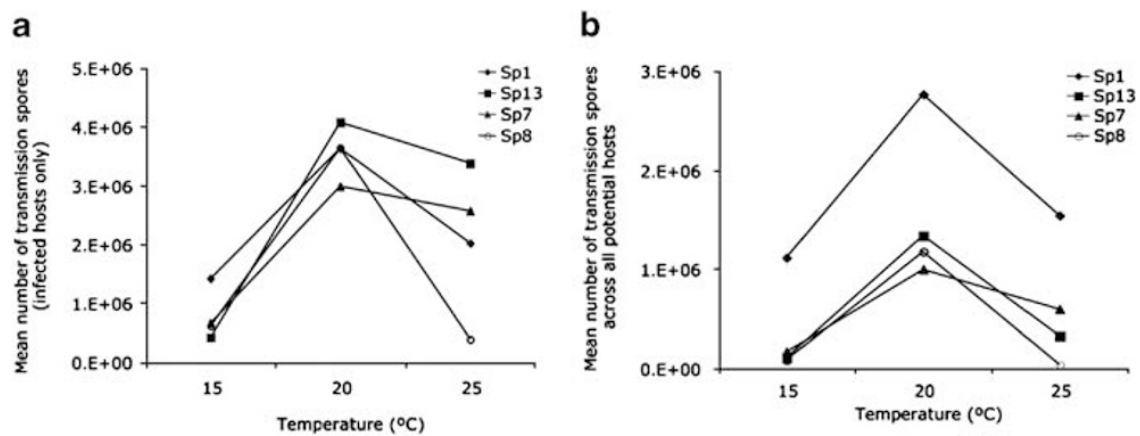

Figure 3 Measures of parasite fitness considering spore production among infected hosts only or incorporating the ability of parasite genotypes to infect. (a) The average number of spores produced during the experiment across all infected hosts is shown for three temperatures (parasite lifetime transmission potential). (b) The number of transmission spores produced across all hosts, regardless of infection status, incorporates information on the infective ability of each parasite genotype. Where infection was not successful, spore production was zero, and hence this measure of parasite fitness provides a measure of the productivity of infection for each parasite genotype, where the number of spores is weighted by the relative infectivity of each parasite genotype across all host genotypes. Error bars are omitted for clarity. See Table 1 for statistical details. 
of polymorphism through $\mathrm{G} \times \mathrm{E}$ is only possible when the contrast in fitness effects is such that genotypes switch their rank order across environmental gradients (Maynard Smith and Hoekstra, 1980). Indeed, a general issue in studies of $\mathrm{G} \times \mathrm{E}$ is that interactions may come about not because of a change in the rank order of genotypes (changes in the mean), but simply because genotypes have unequal variances across environmental treatments. Moreover, in our experiment, the total variation in infectivity due to variation in temperature or $\mathrm{G}_{\mathrm{H}} \times \mathrm{E}_{\mathrm{T}}$ was small (less than 5 or $3 \%$, respectively), which indicates that infectivity is a trait robust to thermal variation, as was recently reported in a plant-pathogen system (Laine, 2007b, 2008). In an earlier study of thermal variation on infection rates in a different population of the Daphnia-Pasteuria system, crossing reaction norms for infectivity were detected across temperature treatments (Mitchell et al., 2005). The strength of $\mathrm{G}_{\mathrm{H}} \times \mathrm{E}_{\mathrm{T}}$ could differ between populations, but additionally, the earlier study incorporated more host genotypes (16 host genotypes across two experiments) than this study, which might increase the power to detect variation explained by $\mathrm{G}_{\mathrm{H}} \times \mathrm{E}$ effects. With respect to the mechanisms that might underlie $\mathrm{G}_{\mathrm{H}} \times \mathrm{E}_{\mathrm{T}}$, infection occurs by ingestion of spores during filtration feeding, and thus $\mathrm{G}_{\mathrm{H}} \times \mathrm{E}_{\mathrm{T}}$ could reflect genotype-specific differences in filtration rate at different temperatures (for example, Hall et al., 2007).

\section{Host mortality}

Host mortality varied considerably across temperature treatments, and the direction and magnitude of this change depended on the parasite genotype $\left(\mathrm{G}_{\mathrm{P}} \times \mathrm{E}_{\mathrm{T}}\right.$, Table 1, Figure $2 b$ ). $G_{P} \times E_{T}$ for mortality of infected hosts will affect the parasite directly, as transmission in this system is exclusively achieved through the release of transmission spores at host death (Ebert et al., 1996). For example, at $20^{\circ} \mathrm{C}$, parasite genotype Sp8 did not kill any of the hosts it infected during 900 degree-days (45 days) (Figure 2b) and produced the least number of spores compared with the other genotypes tested. However, at $25^{\circ} \mathrm{C}$, this situation was reversed; not only did this parasite genotype kill all the hosts it infected, it did so faster than any other parasite genotype (Figure 3a). Results from both demographical and epidemiological models show that selection favours early over late births in expanding populations, so in an epidemic there can be a benefit to early transmission and short generation time even if it lowers the pathogen's lifetime reproduction (Bull and Ebert, 2008). This highlights how more virulent strains might be afforded an evolutionary advantage under some environmental conditions, in this case, increased temperature. Indeed, given that $P$. ramosa epidemics tend to coincide with increases in temperature in the wild (Mitchell et al., 2004; Duncan and Little, 2007), such a harmful parasite genotype would be the most likely to gain prevalence if an increase in mean temperature were to occur. However, as we discuss below, an accurate assessment of parasite fitness must take into account additional fitness correlates.

\section{Parasite growth and measures of parasite fitness}

The number of transmission stages produced is an important parasite fitness component. Once infection was achieved we found that the genotype of the host had no effect on how infections progressed. Instead, our results show that parasite spore production was determined by temperature, and the magnitude and direction of this effect depended on the parasite genotype $\left(\mathrm{G}_{\mathrm{P}} \times \mathrm{E}_{\mathrm{T}}\right.$, Table 2, Figure 3a). This interaction may be due to slightly divergent slopes of the reaction norms rather than due to a change in the rank order of genotypes between treatments. Again, care should be taken when interpreting $G \times E$ interactions where a clear change in the rank order of genotypes is not observed. In this case, however,

the genotype-by-temperature interaction follows earlier reports on the effect of temperature on parasite growth and transmission potential in this (Vale et al., 2008) and other systems (for example, Blanford et al., 2003; Laine, $2007 \mathrm{~b}$ ). We attempted to dissect the causes of variation in the number of spores produced by controlling for differential survival among hosts and thus estimating parasite growth rate. We did not find a $G_{P} \times E_{T}$ interaction for parasite growth rate (Table 2), suggesting that variation in survival substantially contributed to $G_{P} \times E_{T}$ interaction for lifetime transmission potential.

The $G_{P} \times E_{T}$ interaction we observed for lifetime transmission potential indicates the potential for environment-dependent selection to maintain polymorphism in the parasite population (Byers, 2005; Laine and Tellier, 2008). However, producing transmission stages also depends on infecting the host, and our analysis of infectivity revealed a somewhat different set of interactions (in particular, $\mathrm{G}_{\mathrm{H}} \times \mathrm{E}_{\mathrm{T}}$ ) than did our analysis of transmission-stage production (where $G_{P} \times E_{T}$ was more important). Therefore, we were interested in determining if the $G_{P} \times E_{T}$ interaction we observed for differences in transmission-stage spores remained when information about transmission-stage production was combined with infectivity. To achieve this, we considered the average spore production of each parasite genotype on all hosts, regardless of their infection status. This allows for a more complete measure of parasite fitness that incorporates both its probability of infecting a host and growth following infection: hosts that did not become infected contributed zero spores. The $\mathrm{G}_{\mathrm{P}} \times \mathrm{E}_{\mathrm{T}}$ interaction that was significant for lifetime transmission potential among infected hosts (Table 2; Figure 3a) was no longer evident once this information about parasite infectivity was incorporated into parasite fitness (Table 2; Figure 3b). Notably, parasite genotype Sp1 is by far the genotype with highest (estimated) fitness at all temperatures (Figure 3b), even though it was not the genotype producing the highest number of spores (Figure $3 \mathrm{a}$ ) during infections. This is a reflection of it having the highest mean infectivity across all host genotypes (Figure 1) and highlights the importance of infectivity for parasite fitness. These data suggest that variation in the number of transmission stages produced per infection between different parasite genotypes is not large enough to overcome the large differences in their ability to infect. The ability to infect is the strongest determinant of parasite fitness.

This result highlights the importance of considering variation that arises through the natural route of infection. When the natural route of infection is not possible, some studies artificially inject parasites directly into their hosts and measure parasite growth rate only 
(this is common, for example, in Drosophila and in vertebrate models). In this study, ignoring variation in infectivity would have given a very different picture of parasite fitness. Another study measuring parasite local adaptation has also emphasized how using different measures of parasite fitness can lead to different conclusions about trajectories of parasite evolution (Laine, 2008). In some sense, by simultaneously taking infectivity and spore production into account, our measure of parasite fitness is closer to the fitness measures obtained in bacteria-phage systems, where measuring phage population growth rate takes into account both the ability to infect and the growth within the host cell (Abedon, 2008).

However, this does not necessarily mean that the observed $G_{P} \times E_{T}$ interaction for spore production is inconsequential. Earlier studies have shown that the number of $P$. ramosa spores a host is exposed to (that is, dose) strongly influences the likelihood of achieving a successful infection (for example, Ebert et al., 2000; Ben-Ami et al., 2008). Thus, the detection of parasite $\mathrm{G}_{\mathrm{P}} \times \mathrm{E}_{\mathrm{T}}$ interactions for both transmission-stage production and time to host death (the timing of transmission in this system) could essentially change the 'dose' of infection, raising the possibility that subsequent cycles of infection will result in different patterns of infectivity. Testing this hypothesis experimentally, however, may not be straightforward. For example, Ben-Ami et al. (2008) recently tested the relationship between parasite dose and infectivity for 14 host-parasite combinations (seven host genotypes and two parasite genotypes) in a constant environment. Five of these combinations showed a density-dependent relationship between parasite dose and infection levels, and for these combinations, the relationship was found to fit well with a model that assumed the existence of non-inherited phenotypic differences in host susceptibility (phenotypic plasticity). For this reason, predictions about how $G_{P} \times E$ interactions for transmission-spore production will affect infectivity levels in subsequent infection cycles occurring in a variable environment are not intuitively obvious, and will depend on the particular combinations of host and parasite genotypes involved, and on the extent to which hosts are plastic in their responses to infection.

\section{How relevant are $\mathrm{G} \times \mathrm{G} \times \mathrm{E}$ interactions?}

One outcome of this study was that a significant $G_{H} \times G_{P} \times E_{T}$ interaction was not detected for any of the traits we measured. Studies on other systems have found strong $G_{H} \times G_{P} \times E$ interactions (for example, Tetard-Jones et al., 2007), whereas other workers (for example, Heath and Tiffin, 2007; Laine, 2007b) found results similar to the present one: considerable $\mathrm{G} \times \mathrm{E}$ interactions for individual traits, but only weak or insignificant three-way interactions. Thus, the general importance of $\mathrm{G} \times \mathrm{G} \times \mathrm{E}$ remains uncertain; they may only be relevant for particular systems.

One clearly important source of environmental variation concerns differences between the field and laboratory. Work on a snail-schistosome system (Theron et al., 2008), for example, found that patterns of hostparasite compatibility $\left(G_{H} \times G_{P}\right)$ depended substantially on whether individuals were recently field-collected or were longer-term laboratory cultures. In a recent investi- gation of parasite local adaptation (Laine, 2007a), two experiments found discordant results when measuring parasite fitness either in a field-transplant experiment or in a laboratory cross-infection experiment. Such patterns have led some authors to propose that both laboratorybased and field-transplant experiments should be the norm for local adaptation studies (Nuismer and Gandon, 2008), though this certainly will not always be possible. Despite these concerns, the lack of a significant threeway interaction in our experiments has an important implication. $G_{H} \times G_{P}$ interaction indicates the potential for frequency-dependent coevolutionary interactions, and our results suggest that the signal of coevolutionary dynamics in the wild should shine through the noise of thermal variation.

\section{Acknowledgements}

We thank Sarah Hall for technical assistance during the experimental work, Martin Stjernman for thoughtful discussion, and David Shuker and Nick Colegrave for advice on the statistical analyses. Pedro Vale is supported by the GABBA Graduate Program in Areas of Basic and Applied Biology, University of Porto, and by a PhD studentship from Fundação para a Ciência e Tecnologia, Portugal. Tom Little is supported by Wellcome Trust Senior Research Fellowship in Basic Biomedical Sciences.

\section{References}

Abedon ST (2008). Bacteriophage Ecology: Population Growth, Evolution, and Impact of Bacterial Viruses. Cambridge University Press: New York, NY, USA

Bedhomme S, Agnew P, Sidobre C, Michalakis Y (2004). Virulence reaction norms across a food gradient. Proc Biol Sci 271: 739-744.

Ben-Ami F, Regoes RR, Ebert D (2008). A quantitative test of the relationship between parasite dose and infection probability across different host-parasite combinations. Proc Biol Sci 275: 853-859.

Blanford S, Thomas MB, Pugh C, Pell JK (2003). Temperature checks the Red Queen? Resistance and virulence in a fluctuating environment. Ecol Lett 6: 2-5.

Bull JJ, Ebert D (2008). Invasion thresholds and the evolution of nonequilibrium virulence. Evol Applications 1: 172-182.

Byers DL (2005). Evolution in heterogeneous environments and the potential of maintenance of genetic variation in traits of adaptive significance. Genetica 123: 107-124.

Carius HJ, Little TJ, Ebert D (2001). Genetic variation in a host-parasite association: potential for coevolution and frequency-dependent selection. Evolution 55: 1136-1145.

Crawley MJ (2007). The $R$ book. John Wiley: New York.

Duncan AB, Little TJ (2007). Parasite-driven genetic change in a natural population of Daphnia. Evolution 61: 796-803.

Ebert D (1998). Experimental evolution of parasites. Science 282: 1432-1435.

Ebert D, Rainey P, Embley TM, Scholz D (1996). Development, life cycle, ultrastructure and phylogenetic position of Pasteuria ramosa Metchnikoff 1888: rediscovery of an obligate endoparasite of Daphnia magna Straus. Philos Trans $R$ Soc Lond 351: 1689-1701.

Ebert D, Zschokke-Rohringer CD, Carius HJ (2000). Dose effects and density-dependent regulation of two microparasites of Daphnia magna. Oecologia 122: 200-209.

Ferguson HM, Read AF (2002). Genetic and environmental determinants of malaria parasite virulence in mosquitoes. Proc Biol Sci 269: 1217-1224. 
Hall SR, Sivars-Becker L, Becker C, Duffy MA, Tessier AJ, Caceres CE (2007). Eating yourself sick: transmission of disease as a function of foraging ecology. Ecol Lett 10: 207-218.

Hamilton WD (1993). Haploid dynamic polymorphism in a host with matching parasites-effects of mutation subdivision, linkage, and patterns of selection. Heredity 84: 328-338.

Heath KD, Tiffin P (2007). Context dependence in the coevolution of plant and rhizobial mutualists. Proc Biol Sci 274: 1905-1912

Jensen KH, Little T, Skorping A, Ebert D (2006). Empirical support for optimal virulence in a castrating parasite. PLoS Biol 4: e197.

Kaltz O, Shykoff JA (1998). Local adaptation in host-parasite systems. Heredity 81: 361-370.

Kluttgen B, Dulmer U, Engels M, Ratte HT (1994). An artificial fresh-water for the culture of zooplankton. Water Res 28: 743 .

Laine AL (2007a). Detecting local adaptation in a natural plantpathogen metapopulation: a laboratory vs field transplant approach. J Evol Biol 20: 1665-1673.

Laine AL (2007b). Pathogen fitness components and genotypes differ in their sensitivity to nutrient and temperature variation in a wild plant-pathogen association. J Evol Biol 20: $2371-2378$.

Laine AL (2008). Temperature-mediated patterns of local adaptation in a natural plant-pathogen metapopulation. Ecol Lett 11: 327-337.

Laine AL, Tellier A (2008). Heterogeneous selection promotes maintenance of polymorphism in host-parasite interactions. Oikos 117: 1281-1288.

Lambrechts L, Chavatte JM, Snounou G, Koella JC (2006a). Environmental influence on the genetic basis of mosquito resistance to malaria parasites. Proc Biol Sci 273: 1501-1506.

Lambrechts L, Fellous S, Koella JC (2006b). Coevolutionary interactions between host and parasite genotypes. Trends Parasitol 22: 12-16.

Lambrechts L, Halbert J, Durand P, Gouagna LC, Koella JC (2005). Host genotype by parasite genotype interactions underlying the resistance of anopheline mosquitoes to Plasmodium falciparum. Malar J 4: 3.

Lazzaro BP, Little TJ (2008). Review. Immunity in a variable world. Philos Trans Biol Sci 364: 15-26.

Lazzaro BP, Sackton TB, Clark AG (2006). Genetic variation in Drosophila melanogaster resistance to infection: a comparison across bacteria. Genetics 174: 1539-1554.
Maynard Smith J, Hoekstra R (1980). Polymorphism in a varied environment: how robust are the models? Genet Res 35 45-57.

Mitchell SE, Read AF (2005). Poor maternal environment enhances offspring disease resistance in an invertebrate. Proc Biol Sci 272: 2601-2607.

Mitchell SE, Read AF, Little TJ (2004). The effect of a pathogen epidemic on the genetic structure and reproductive strategy of the crustacean Daphnia magna. Ecol Lett 7: 848-858.

Mitchell SE, Rogers ES, Little TJ, Read AF (2005). Host-parasite and genotype-by-environment interactions: temperature modifies potential for selection by a sterilizing pathogen. Evolution 59: 70-80.

Nuismer SL, Gandon S (2008). Moving beyond common-garden and transplant designs: insight into the causes of local adaptation in species interactions. Am Nat 171: 658-668.

Peters RH, De Bernardi R (1987) Daphnia. Memorie Dell 'Istituto Italiano di Idrobiologia.

R (2005). R: A Language and Environment for Statistical Computing. $\mathrm{R}$ Foundation for Statistical Computing: Vienna, Austria.

Read AF, Schrag SJ (1991). The evolution of virulence: experimental evidence. Parasitol Today 7: 296-297.

Restif O, Kaltz O (2006). Condition-dependent virulence in a horizontally and vertically transmitted bacterial parasite. Oikos 114: 148-158.

Salvaudon L, Héraudet V, Shykoff J (2007). Genotype-specific interactions and the trade-off between host and parasite fitness. BMC Evol Biol 7: 189.

SAS Institute Inc. C., NC. JMP 7 in 1989-2007, ed, Cary, NC.

Tetard-Jones C, Kertesz MA, Gallois P, Preziosi RF (2007). Genotype-by-genotype interactions modified by a third species in a plant-insect system. Am Nat 170: 492-499.

Theron A, Coustau C, Rognon A, Gourbiere S, Blouin MS (2008). Effects of laboratory culture on compatibility between snails and schistosomes. Parasitology 135: 1179-1188.

Thompson JN (1994). The Coevolutionary Process. University of Chicago Press: Chicago.

Thompson JN (1999). Specific hypotheses on the geographic mosaic of coevolution. Am Nat 153: S1-S14.

Thompson JN, Burdon JJ (1992). Gene-for-gene coevolution between plants and parasites. Nature 360: 121.

Vale PF, Stjernman M, Little TJ (2008). Temperature dependent costs of parasitism and the maintenance of polymorphism under genotype-by-environment interactions. J Evol Biol 21: 1418-1427. 